\title{
Enhancing Quality of MPEG Video through Partially Reliable Transport Service in Interactive Application
}

\author{
Vincent Lecuire ${ }^{1}$, Francis Lepage ${ }^{1}$, and Khalil Kammoun ${ }^{2}$ \\ ${ }^{1}$ Centre de Recherche en Automatique de Nancy (CNRS ESA 7039), \\ BP 239, F-54506 Vandoeuvre-lès-Nancy, France \\ \{Vincent.Lecuire, Francis.Lepage\}@cran.uhp-nancy.fr \\ ${ }^{2}$ University of Karlsruhe, Kaiserstr. 12, \\ D-76128 Karlsruhe, Germany
}

\begin{abstract}
Real-time video communication over a packet switching network is subject to losses and errors. In few networking environment, a partially reliable transport service based on error control by retransmission is a possible option. We propose a retransmission-based correction scheme which is driven by the time remaining for the data delivery and also by the importance of artifacts the data loss produces in the reconstructed video. This scheme is applied to continuous MPEG video packet streams. Experimental results show that the picture quality of MPEG video is degraded more gracefully in the presence of network errors, and those with a low cost on network bandwidth. In addition, the best QoS policy to use the partially reliable transport service is highlighted.
\end{abstract}

\section{Introduction}

Real-time compressed video stream over a packet switching network is very sensitive to losses and errors. Network errors require the use of error correction and concealment techniques to provide the video quality necessary for the video based applications to be widely accepted and used. In general, error correction by retransmission is considered as not useful because video data must be played back continuously with a short delay time. In addition, when errors are due to network congestion, the retransmission of lost packets is not the correct reaction because it aggravates the situation and jams the network. Nevertheless, when time driven retransmission can be performed with a high probability of success, a retransmission-based approach becomes a viable option for error control on real-time stream, as is shown in [5] for audio, and in [20] for video. In comparison with forward error correction (FEC) which is very costly in extra bandwidth $[18,19]$, correction by retransmission can be very attractive under the condition that the number of retransmissions is well controled.

In this paper, we propose a novel retransmission-based error control scheme in order to provide a partially reliable transport service suitable for interactive video application. Retransmission is driven by the time remaining for the data delivery, as well as 
the importance of artifacts the data loss produces in the reconstructed video. A such transport service is attractive for error control on video coded with motion compensation techniques where losses in certain parts of the data stream are more annoying when viewed than losses in other parts of the data stream. Also, our scheme is applied to the MPEG coded video. In the following, section 2 presents the special features of our retransmission-based scheme in framework of an unicast transport protocol. Section 3 briefly describes the MPEG standard and evaluates the impact of losses on the coded MPEG video packet stream. The most and the least important parts of coded video packet stream are highlighted for partially reliable transport service requirements. In section 4, the experimental results are shown and discussed with an accompanying analysis. This shows the error control policy which is the best in the ratio between the picture quality improvement and the network bandwidth cost. Finally, section 5 concludes with a discussion of the possible extensions for the proposed service and future research developments.

\section{An Error Control Scheme for Partially Reliable Transport Service}

In this section, we present a partially reliable transport service, so that application's delay and error requirements are best respected. Few error control schemes for a such transport service have been yet proposed in $[5,7,16,6,8]$. However, the novel error control scheme that we propose is attractive in interactive multimedia application because application can specify the packet's QoS on the fly of the packetized stream.

\subsection{Motivations}

Our error control scheme, TSR, is based on retransmission which is driven by the time remaining for the data delivery and also by the packet's QoS class. This scheme is original due to QoS driven retransmission, which distinguishes two kinds of data packets :

- valuable packets (v-packets) : The loss of these packets is judged as serious for the application, and the retransmission should be tried if the time remaining until its scheduled playout time is more than one network round trip time.

- ordinary packets (o-packets) : The loss of these packets is considered as tolerable for the application, and the data transfer should proceed on as if nothing happens.

For requirements of interactive multimedia applications, we assume an error control that is characterized by two main features :

- Firstly, we give the responsibility of the error correction to the receiver side. In this case, the receiver returns to sender a negative acknowledgment (NAK) in order to request the retransmission of lost v-packets. The NAK approach is the best for the bandwidth cost to be added by the error correction scheme in comparison to cumulative acknowledgment (ACK) approach : With the ACK approach in effect, when an ACK message is lost, the sender will execute many useless retransmissions. 
- Secondly, the QoS class associated with a data packet is attributed by the application to the sender side. It is absolutely necessary to prepare an on-line, and finegrained QoS specification of all data units, which are marked on the fly of the packet stream building by the application. This feature is well suitable with the ALF (Application Level Framing) architectural concept [4].

The set of these two features conduces to a tricky challenge : When a packet is lost by the network, the receiver must to be able, both to detect this loss and also to identify its QoS class in order to launch a correct reaction. We treat this problem by using a technique for lasting coloration of data packets.

\subsection{Technique for Lasting Coloration of Packets}

This technique was presented in detail in [13]. Its principle consists in packet tagging, depending on the QoS class of the contained data, and with a lasting property in order to be recoverable without the packet itself. Informally, we refer to this tagging as the lasting coloration given to the packet.

\subsubsection{Tagging the Packet Coloration at Sender Side}

The coloration is tagged directly in the data packet by a packet numbering using a pair of counters $(i, j)$. The counter $i$ is used to trace the evolution of the v-packet sequence ; the counter $\mathrm{j}$ serves to trace the evolution of the o-packet sequence. However, this sequence number is not used for the usual sequence number because the roles are different. The packet numbering technique is shown in Fig. 1 with any QoS policy for partially reliable service.

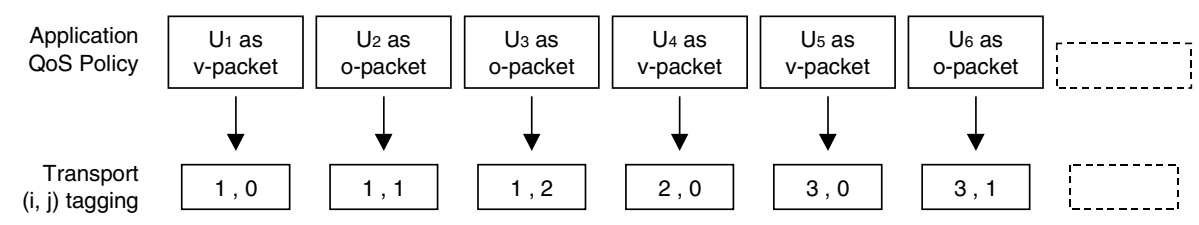

Fig. 1. A sample of $(i, j)$ numbering for coloration of packets. The QoS class which is affected to a data packet is carried on the counter $\mathrm{j}$. When $\mathrm{j}$ is equal to 0 , it is a valuable data packet ( $\mathrm{v}$ packet). When $\mathrm{j}$ is greater than 0 , it is a ordinary data packet (o-packet).

The sequence number $(i, j)$ evolves according to the following rules:

- $\mathrm{i}$ and $\mathrm{j}$ are cyclical ordinal counters, with $0 \leq \mathrm{i}<\mathrm{k}$ and $0 \leq \mathrm{j}<\mathrm{p}$, where $(\mathrm{k}, \mathrm{p}) \mathrm{de}-$ fines the dimension of the numbering range (by example $\mathrm{k}=\mathrm{p}=2^{32}$ ).

- The associated packet color is carried on the counter $j, j=0$ indicating a v-packet while $\mathrm{j} \neq 0$ an o-packet.

- $\mathrm{i}$ and $\mathrm{j}$ are initialized both to 0 at the connection establishment.

- When the application requests to transmit a v-packet, $i$ is incremented and $j$ is reseted in the general case. Assuming this one follows $(i, j)$ packet, it is numbered $(i+1,0)$ if $i+1<k$, or $(0,0)$ if $i+1=k$. 
- When the application requests to transmit an o-packet, $\mathrm{j}$ is incremented in the general case. Assuming this one follows $(i, j)$ packet, it is numbered $(i, j+1)$ if $j+1<p$. But it is numbered $(i+1,0)$ if $j+1=p$ and $i+1<k$, or $(0,0)$ if $j+1=p$ and $i+1=k$. The coloration of the packet is forced to the v-packet by the protocol in these both last cases. More the $\mathrm{k}$ and $\mathrm{p}$ values will be big, more a such situation will be rare.

\subsubsection{Identifying the Coloration at Receiver Side}

On the receiver side, the coloration of the lost packet should be identified to launch a correct reaction. A proper functioning requires the detection of all v-packet losses in order to test this correction. This is the case with the proposed numbering technique. In the following, we assume the last packet received correctly is numbered $(1,0)$, as that is shown in Fig. 2. The next expected packet would have a number that depends on its color : the number $(1,1)$ for a o-packet, or $(2,0)$ for an v-packet. In one case the packet $(1,1)$ is delivered is normal. In a second case of the packet $(2,0)$ also, but an ambiguity could not be resolved by the receiver if the loss of o-packet series beginning with $(1,1)$ occured. Finally, the arrival of a packet having a number different from $(1,1)$ and $(2,0)$ presents a third case, where the packet loss can be detected. A comparison between the two last packet numbers allows the determination of the lost packet color : If the counter $i$ has not changed, the loss has affected only o-packets ; in the other case, where the counter i has changed, the loss affects v-packets.

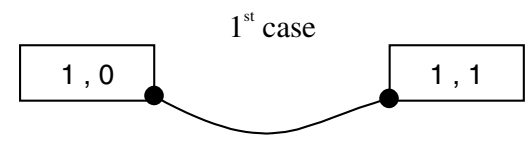

No gap $\rightarrow$ OK

$2^{\text {nd }}$ case

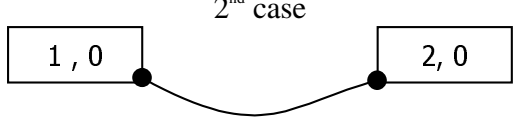

No gap $\rightarrow$ OK

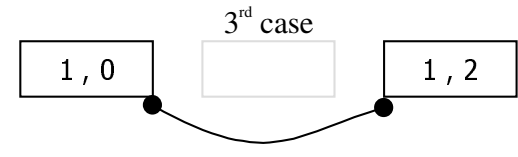

(a) Gap : Counter i has not changed $\rightarrow$ o-packet lost only

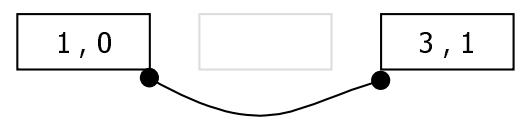

(b) Gap : Counter i has changed $\rightarrow$ v-packet lost

Fig. 2. Identification of its lasting coloration when packet is lost.

This original lasting coloration tagged directly in the packet guarantees the detection by the receiver of any v-packet loss, and allows the detection of most o-packet losses. This lasting coloration given to each packet, indicates how it needs to be controlled for its loss. This is a deterministic error correction scheme.

\subsection{Technique for Time Driven Retransmission}

In time driven retransmission part, the scheme is classic, based on the playout delay initialization, the packet timestamp, and the network delay and jitter estimation. The playout delay must to be adjusted in order to compensate for variable network delays, 
and also to give extra time to attempt some correction. As in [17], the playout delay is defined to be the difference between the playout time for the receiver and the generation time for the sender. Various studies concluded that for natural hearing, interactive real-time applications can tolerate a latency of about $250 \mathrm{~ms}$ for only video stream [9], and of about $100 \mathrm{~ms}$ for audio/video stream [12]. Received packets are first queued into a smoothing buffer until their scheduled playout. When a loss of packet is detected on the side of the receiver, its retransmission can be requested if the delay till its scheduled playout is more than one round trip time (RTT).

\subsection{Global Protocol Building}

We have implemented a connection oriented transport protocol which is based on TSR scheme. This transport protocol (TSR-TP) has been designed as a light-weight protocol working on the top of UDP/IP. It is characterized as below :

- A three-way handshake procedure is used to establish a TSR-TP connection. In this step, network delay is estimated for playout delay auto-adjustement.

- NAK strategy is used to request packet retransmission applying selective repeat policy. The NAK message contains information including the part $\mathrm{i}$ in $(\mathrm{i}, \mathrm{j})$ number of the last v-packet that has been received in sequence and such a 64-bits value representing the table of the 64 next v-packets. One bit is set to 1-value in order to indicate that the corresponding v-packet in the table must be retransmitted. Inversely, one bit is reset to 0 -value when the corresponding $v$-packet has been received successfully by the receiver.

- In a similar way to RTCP, report is periodically generated by the receiver to convey feedback on quality of data delivery and information of sender. The report message contains information including the highest packet number received, the number of packets lost, and timestamp. In the experiments described below, the delay between a report and the following is configured to $250 \mathrm{~ms}$ (i.e. 2 per GOP).

TSR-TP provides a partially-reliable transport service, i.e less-than-fully reliable and more-than-no reliable data transfer. That is akin to the concept of partial order connection (POC) presented in [1]. In this viewpoint, TSR-TP can be considered as an example of POC.

\section{MPEG over Best-Effort Network}

This section shows that the MPEG coding is well adapted to the partially reliable transport service which has been presented in previous section when this video stream is transmitted over best-effort network.

\subsection{MPEG Video Coding}

The MPEG-1 (Motion Picture Experts Group) video encoding standard [11] was designed to support video encoding at bit rates of approximately $1.5 \mathrm{Mbps}$. The image 
size is based on the QIF-format (352x288 pixels). The quality of the video achieved with this standard is rough, but is good enough for person-to-person audio/video interaction or video-aided remote control. MPEG-2 was developed to meet the demands for high quality video coding. It is similar to MPEG-1. The MPEG compression algorithm relies on two basic techniques : block-based motion compensation (MC) for the reduction of the temporal redundancy and Discrete Cosine Transform (DCT) based compression for the reduction of spatial redundancy [14].

Three types of compressed frames are generated by an MPEG encoder : Intra-coded (I), Predictive (P), and Bi-directional (B) frames. The use of these three frame types allows MPEG to be robust (I-pictures provide error propagation reset points) and efficient (B- and P-pictures allow a good overall compression ratio). An I-frame is encoded independently from other frames based on DCT coding. A P-frame is encoded with reference to a past I- or P-frame, and is used as a reference point to the next P-frame. A B-frame is an interpolated frame that requires both a past and a future reference frame (I or P), and results in the highest amount of compression. B frames are never used as reference frames.

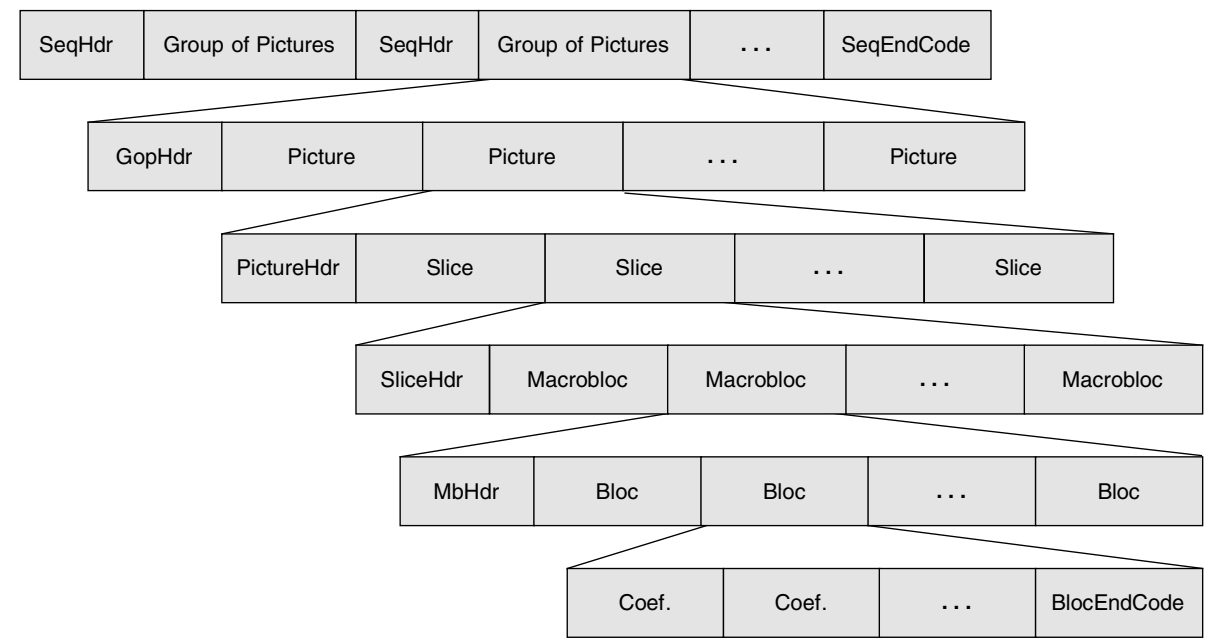

Fig. 3. An MPEG video stream is highly hierarchically structured, with six layers : Block, macroblock, slice, picture, GOP, and sequence. Each layer supports a definite function : Either a signal-processing function (Discrete Cosine Transform, Motion Compensation) or a logical function (Resynchronization, Random access point).

The MPEG video bitstream is hierarchically structured as illustrated in Fig. 3. The smallest entity defined by the standard is the block, which is an area of 8x8 pixels of luminance or chrominance. A macroblock contains four blocks of luminance samples and two, four or eight blocks of chrominance samples, depending on the chrominance format. A variable number of macroblocks is encapsulated in an entity called slice. A picture is composed of a variable number of slices. The entity called group of pictures (GOP) defines the relative frequency of occurrence of I, P, and B frames in the sequence. The application can fixe the encoding pattern by specifying the value for both 
of $\mathrm{M}$, the distance between successive $\mathrm{P}$ frames, and $\mathrm{N}$, the distance between successive I frames. A I-frame (one per GOP) is always placed at the beginning of the GOP and followed by a number of P-frames, which are interspersed between B-frames. Each frame contains exactly one picture.

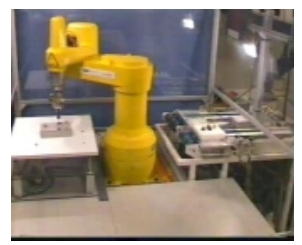

$\mathrm{I}_{0}-\mathrm{GOP} 1$

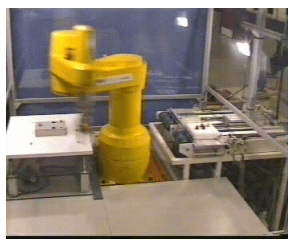

$\ldots \mathrm{P}_{9} \ldots$

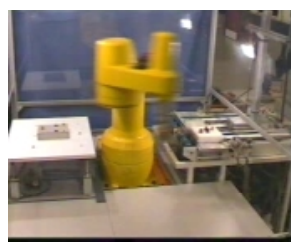

$\mathrm{I}_{0}-\mathrm{GOP} 2$

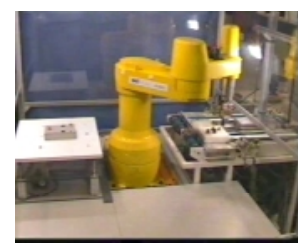

$\ldots \mathrm{P}_{6}(\mathrm{GOP} 2) \ldots$

Fig. 4. The video robot sample used for experimentations. Encoding parameters value $\mathrm{N}=12$, $\mathrm{M}=3$, and $\mathrm{S}=18$. The GOP is structured as I0-B1-B2-P3-B4-B5-P6-B7-B8-P9-B10-B11.

\subsection{The Video Sample Used for Experimentations}

All the experiments are executed in this paper on an two-minute long MPEG-1 elementary video stream with data constant bit rate encoding of $1 \mathrm{Mbps}$, for a total size of 16,8 Mbyte. The video sample shows a manufacturing robot in operation, as shown in Fig. 4. The scene was shot in a single fixed plan. The table 1 provides the statistics on the MPEG robot sample.

Table 1. Statistics for video robot sample.

\begin{tabular}{ccccc}
\hline Frame & \multicolumn{2}{c}{ Frames } & \multicolumn{2}{c}{ Bytes } \\
Type & nr. & $\%$ & nr. & $\%$ \\
\hline I & 251 & 8.4 & 3430184 & 19.5 \\
B & 750 & 25.0 & 5945588 & 33.8 \\
P & 2000 & 66.6 & 8223618 & 46.7 \\
\cline { 2 - 2 } Total & 3001 & & 17599390 & \\
\hline
\end{tabular}

\subsection{Network Loss Effects on MPEG Video Data}

In an MPEG video stream, packet loss by the network reduces quality depending strongly on the type of the lost information [2]. Losses of syntactic data, such as headers and system information, affect the quality differently than losses of semantic data such as pure video information (e.g. motion vectors, DCT coefficients, etc.). Their effects on video quality are studied in detail in [3]. The MPEG sequence header information is very important in configuring system and computation environment. The picture header contains crucial information to initiate and configure the decoder for picture stream decoding. The GOP header provides a resynchronization point when errors occur. However, the GOP header is not so important as the sequence header and the picture header : Indeed if the GOP header is lost, there is no effect on the video quality. 


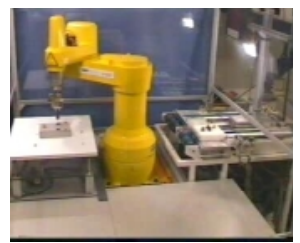

$\mathrm{I}_{0}-\mathrm{GOP} 1$

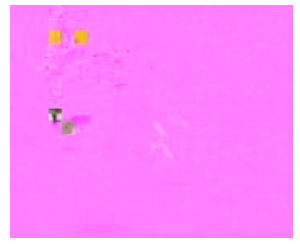

$\ldots \mathrm{P}_{6} \ldots$

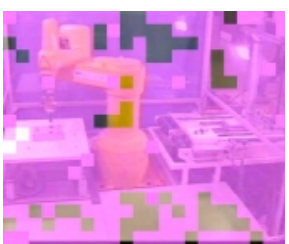

$\ldots \mathrm{B}_{1} \ldots$

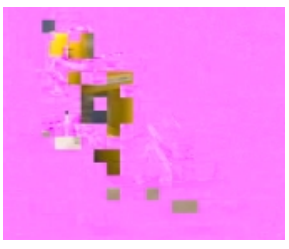

$\ldots \mathrm{P}_{9} \ldots$

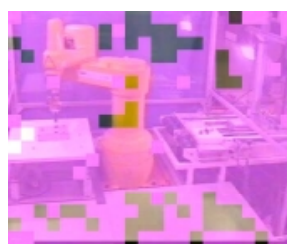

$\ldots \mathrm{B}_{2} \ldots$

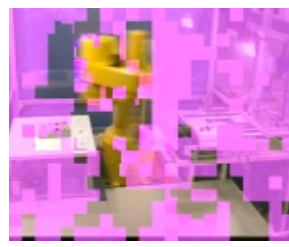

$\mathrm{B}_{11} \ldots$

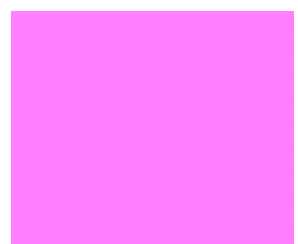

$\ldots \mathrm{P}_{3} \ldots$

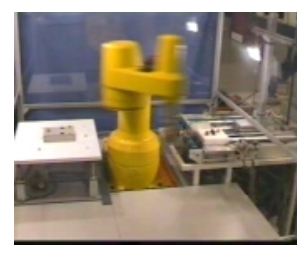

$\mathrm{I}_{0}-\mathrm{GOP} 2$

Fig. 5. Impact of the loss of the picture header on $\mathrm{P}_{3}$. It seriously disrupts the decoding process and leads to the loss of the original picture. Since $\mathrm{P}_{3}$ is used as a reference picture, it affects also the predicted pictures, which reference the $\mathrm{P}_{3}$ 's macroblocks.

Furthermore, quality reduction depends on the location of the lost semantic data due, not only to the predictive structure of MPEG video coded streams, but also to the visual relevance of the data. Data loss spreads within a single picture up to the next resynchronization point (e.g. picture or slice headers) mainly due to the use of differential coding, run-length coding and variable length coding. This is referred to as spatial propagation and may damage any type of picture. When loss occurs in a reference picture (intra-coded or predictive frame), the damaged macroblocks will affect the non intra-coded macroblocks in subsequent frame(s), which reference the errored macroblocks. This is known as temporal propagation and is due to inter-frame predictions. Fig. 5 shows an example of network loss effect on the robot sample.

\subsection{Packetization Scheme to Transport MPEG Video Stream}

A well-designed packetization scheme may ease the influence of the packet loss on the picture quality. Some recommendations are suggested in [10] about the packetization to transport video or audio flow over transport protocols supported by RTP. For example, each header must be completely constrained within the packet. In addition, the packetization scheme must take into account the Maximum Transfer Unit (MTU) of the network to bind the maximum packet size. Packets larger than MTU size may be divided into fragments. If any of the fragments is lost during the network transmission, then the entire packet is lost. It is therefore expected that packets larger than MTU size will have a higher packet loss rate than packets smaller than the MTU size. Our MPEG frames are fragmented, respecting the following rules :

- Individual slices from I- and P- frames were put in their own packet. This requirement insures that the beginning of the next slice, after a packet loss, can be found without the receiver having to scan the packet contents for headers. 
- Since B-frame slices are small, and their loss is less noticed, it would be inefficient to pack B-frame slices individually. Also, as far as it is possible in relation to the MTU of the network, we placed several slices from B- frames into a single packet.

- Picture headers were packed at the beginning of the packet enclosing the first slice of the frame they prededed.

- Sequence headers and GOP headers were included in the same packet as the picture header of the I- frame which they preceded.

The packetization scheme takes advantage of the MPEG video stream structure to reduce dependencies among packets and to maximize the amount of decodable data at the receiver end in case of packet losses. It contributes to improve the robustness of the MPEG video.

\subsection{Packet Classification by QoS Order}

Due to the nature of MPEG video data streams, network losses in certain packets are more annoying when viewed than losses in other packets of the data stream. When the packetization scheme is used to transport the MPEG encoded bitstream, a packet error sensitivity list can be established to provide useful information for error control purposes. This list, which is given in table 2, shows a priority list in order to control the losses by QoS driven retransmission.

Table 2. Packet loss sensitivity list.

\begin{tabular}{ccccc}
\hline Rank & $\begin{array}{c}\text { Pck } \\
\text { Type }\end{array}$ & Enclosed Data & \multicolumn{2}{c}{ Error Propagation } \\
\hline 1 & A & Sequence Header & all pictures & Temporal \\
\hline 2 & B & $\begin{array}{c}\text { I- Picture Header } \\
\text { P- Picture Header }\end{array}$ & all slices & $\begin{array}{c}\text { all pictures in GOP } \\
\text { multiple pict. in GOP }\end{array}$ \\
\hline 3 & C & I- Slice & one slice & all pictures in GOP \\
\hline 4 & D & P- Slice & one slice & multiple pict. in GOP \\
\hline 5 & E & $\begin{array}{c}\text { B- Picture Header } \\
\text { B- Slice }\end{array}$ & $\begin{array}{c}\text { all slices } \\
\text { one or more slices }\end{array}$ & one picture \\
\hline
\end{tabular}

A good policy in order to drive a partially reliable transport service is to try not too much correction (i.e. only for the loss concerning the most important packets in term of error sensitivity). The table 3 provides the packet statistics on the MPEG robot sample when the packetization scheme is applied. 


\section{Experimental Results and Discussion}

In this section, the partially reliable transport service is evaluated for MPEG video stream. Several application QoS policies are tested. Results demonstrate a good ratio between the gain of video quality and the cost of network bandwidth.

Table 3. Statistics for packetized video sample.

\begin{tabular}{ccccccc}
\hline Packet & \multicolumn{2}{c}{ Packets } & \multicolumn{4}{c}{ Packet Size } \\
Type & nr. & $\%$ & avg. & $\min$ & $\max$ & total \\
\hline A & 251 & 1.0 & 614 & 584 & 628 & 153992 \\
B & 750 & 3.0 & 326 & 180 & 488 & 244816 \\
C & 4267 & 17.2 & 768 & 260 & 1436 & 3276192 \\
D & 13101 & 52.7 & 435 & 64 & 896 & 5700772 \\
E & 6504 & 26.1 & 1264 & 256 & 1368 & 8223618 \\
\hline
\end{tabular}

\subsection{Description of the Experiments for Performance Evaluation}

For data gathering, we have carried out many experiments. The architecture of the environment is depicted in Fig. 6 : The sender and the receiver use TSR-TP, as described in the section 2 . On the sender side, the program controls the target bit rate for transmission of the video sample, 1 Mbps. Packets exchange between both sender and receiver systems go accross the lossy router which introduces ramdom packet losses and articifial delays, in a same way as well-known dummynet.

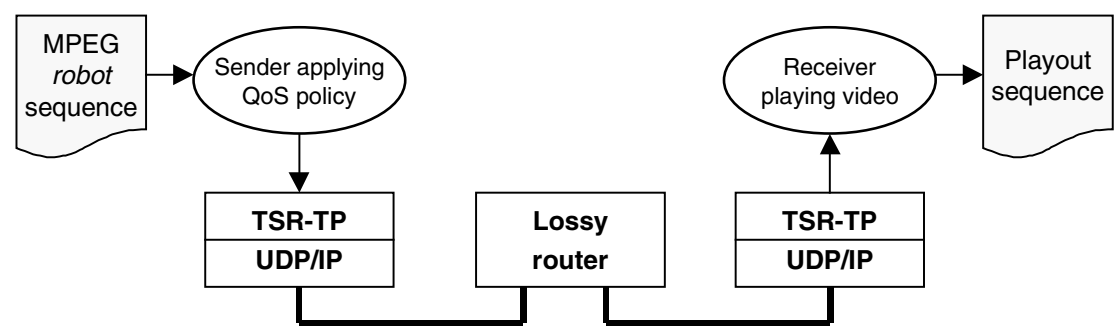

Fig. 6. The experimental environment is based on three computerized systems : The sender, the receiver, and the lossy router.

This experiment has been conducted with the robot sample for variable network loss rate. The average network delay and maximal jitter introducted by lossy router are respectively configured to $25 \mathrm{~ms}$ and $5 \mathrm{~ms}$. In this network condition, we assume that the POC can to attempt only one retransmission when the playout delay is initialized to $100 \mathrm{~ms}$. In order to observe the performance of the POC using the proposed selective packet correction, the sequence has been transmitted with five different application QoS policies : 
- PACK : The video stream is only packetized. The QoS policy is to specify all packets as o-packets. Then none packet is corrected by transport protocol when loss occurs. In this case, the POC provides a full-unreliable transport service.

- SPC1 : Here, the correction must be tried for the most annoying losses - i.e. those which cause an important degradation in the video quality. The QoS policy is to specify packets of type A and B as v-packets, and the others as o-packets. On the robot sample, the POC provides a partially reliable transport service, and $4 \%$ of packets are under the error control.

- SCP2 : Here, the application is more demanding than previously. The QoS policy is to specify packets of type A, B and C as v-packets, for $21 \%$ of packets being under the error control.

- SPC3 : With error concealment technique, MPEG replace a damaged slice in Ppicture with the spatially corresponding slice of the first P- picture of the GOP. For this reason, first $\mathrm{P}$ - picture in the GOP is more important than other $\mathrm{P}$ - picture. So, the QoS policy is to specify packets of type A, B, C as v-packets, more the ones of type D which enclose slices of the first P- picture of each GOP. In the case of this POC, $39 \%$ of packets are under the error control.

- SPC4 : The QoS policy to be evaluated is to specify packets of type A, B, C and D as v-packets. In the case of this POC, $74 \%$ of packets are under the error control.

The experimental result is based both on objective QoS parameters extracted from real-time capture video traces as well as on subjective QoS viewed on the video client. For each experiment run, we computed the average PSNR for all pictures in the video sequence. In addition to assessing the subjective QoS perceived by the end user, 17 users watch the video signal decoded on the video client in all the traffic scenarios. The video material is assessed according to ITU BT.500-7 methodology. With this methodology, the user grades the video material in a 5-value impairment scale : Imperceptible (5), perceptible but not annoying (4), slightly annoying (3), annoying (2), and very annoying (1).

\subsection{Gain of Picture Quality vs. Cost of Bandwidth Quantity}

The gain is measured with a different transmission realization as a function of the packet loss rate, as shown in Fig. 7. It can be seen that the partially reliable service at the transport level enhances the packetization at the application level, achieving graceful degradation as the packet loss probability increases. With the SPC1 policy, the gain of PSNR is low. However, by the correction of I- and P- picture header SPC1 policy allows to rebuild more pictures at the receiver side (2755 vs 2558 with the robot sample of 3001 pictures when the packet loss rate is equal to $12 \%$ ). The loss of a picture is not noticed in the results because in this case, the PSNR is calculated with previous picture. In the cases of SPC2, SPC3 and SPC4 policies, the improvement on the average PSNR is more important. The results of the subjective tests confirm the general idea of an improvement in the video quality. 


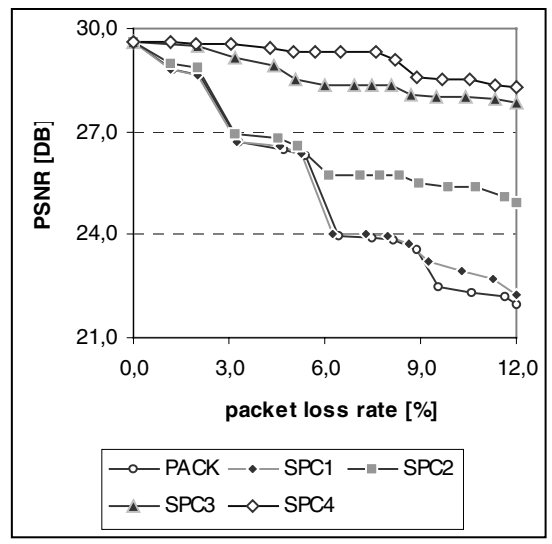

(a)

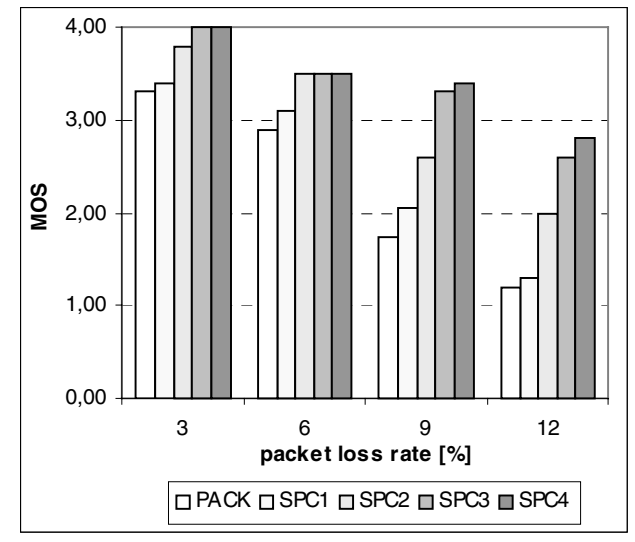

(b)

Fig. 7. Gain of picture quality. Part (a) shows the results of the objective tests. The largest gain is equal to $0.30 \mathrm{~dB}$ for SPC1, $2.98 \mathrm{~dB}$ for SPC2, to $5,58 \mathrm{~dB}$ for SPC3, and to $6.36 \mathrm{~dB}$ for SPC4 when the network loses $12 \%$ of packets. Part (b) shows the results of the subjective tests. The policies under test contribute to improvement respectively until 0.1 point for SPC1, 0.8 point for SPC2, 1.4 point for SPC3, and 1.6 point for SPC4 in the score given by human viewers.

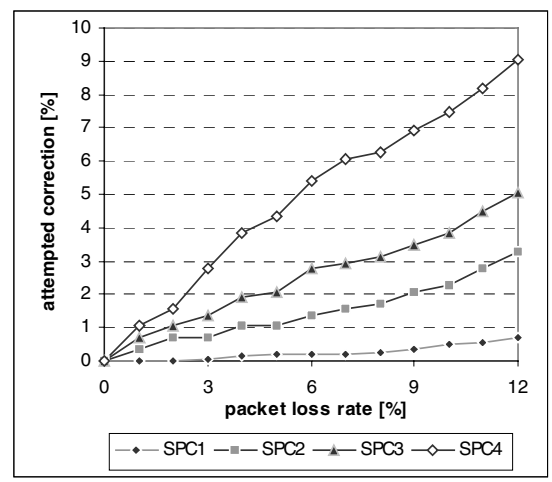

(a)

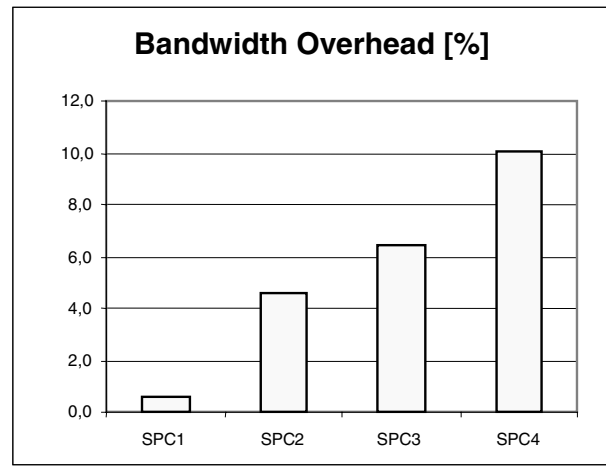

(b)

Fig. 8. Cost of bandwidth quantity. Part (a) shows the percentage of retransmissions which are attempted depending on packet loss rate and the QoS policy applied by application. Part (b) shows the extra bytes imposed by retransmissions. When packet loss rate is equal to $12 \%$, the overhead is to $0.5 \%$ for SPC1, to $4.6 \%$ for SPC2, to $6.4 \%$ for SPC3, and to $10.1 \%$ for SPC4.

The partially reliable transport service which is based on TSR scheme, provides a significant gain of picture quality by well-selected error correction by retransmission. In return, it imposes overhead on network bandwidth which must be evaluated.The results are shown in Fig. 8. We can see that the TSR scheme is not very costly to the network bandwidth in comparison to the use of FEC-based scheme such as Priority Encoding Transmission [15]. Although the performance gains entailed are notable, this FEC-based approach introduces a traffic overhead of about $25 \%$. 


\subsection{Toward a Proposal of the Best QoS Policy}

In order to achieve the comparison between the tested QoS policies for the TSR-based partially reliable transport of interactive MPEG video, our purpose is to establish if it is better to define many of packets as v-packets (i.e. to address the picture quality) than define many of packets as o-packets (i.e. to address the network availability). We have adopted as criteria the ratio between the cost of the retransmissions and the gain of picture quality. On this criteria for comparison, the results show clearly that the SPC3 QoS policy is the best, as depicted in Fig. 9.

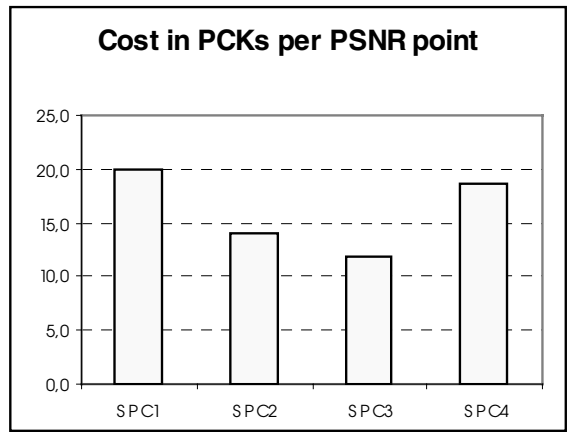

(a)

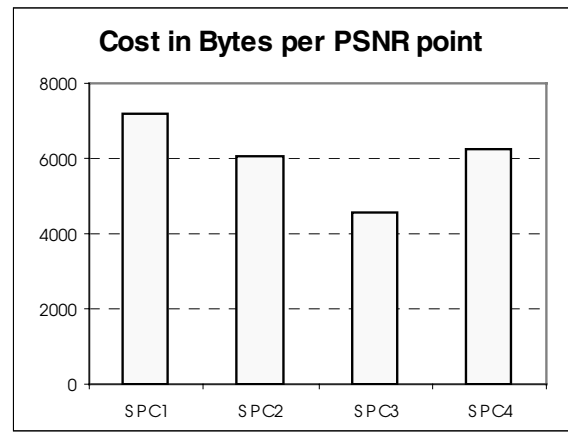

(b)

Fig. 9. Cost per PSNR point. Part (a) shows that TSR-TP generates an average 12 extra packets in order to gain 1 point of PSNR when SPC3 is applied. It is better than with SPC1 (20 extra packets), with SPC2 (14 extra packets), and with SPC4 (19 extra packets). Part (b) conduces to similar results. In order to gain 1 point of PSNR, TSR-TP generates an average 4537 extra bytes when SPC3 is applied. It is better than with SPC1 (7180 extra bytes), SPC2 (6043 extra bytes), and SPC4 (6277 extra bytes).

\section{Conclusion}

In this paper, we have proposed a partially reliable transport service based on time and QoS driven retransmission scheme in order to achieve a deterministic error control. We have shown that this scheme is very suitable when all data packets in the transport packet stream have not the same importance for the QoS perceived by the end user, as this is the case in MPEG video packetized stream. When the use of a such partially reliable service is possible, this approach is attractive because it imposes little overhead on network resources, as that has been resulted from our performance evaluation executed by experimentations. This experiment has highlighted the best QoS policy for MPEG video based application, on the criteria of ratio between the picture quality improvement and the network bandwidth cost. Finally, a perspective of this work is to show that TSR-TP is suitable on MPEG-4 as well as JPEG2000 coded video streams. 


\section{References}

1. Amer, P., Chassot, C., Connolly, C., Conrad, P., Diaz, M.: Partial Order Transport Service for Multimedia Applications. IEEE/ACM Transactions on networking, vol. 2, no. 5 (1994)

2. Boyce, J.M., Gaglianello, R.: Packet Loss Effects on MPEG Video Sent Over the Public Internet. Proc. of ACM Int. Multimedia Conference, Bristol, England (1998)

3. Chiu, D., Cai, L., Mc Cutcheon, M., Ito, M., Neufeld, G.: Transport of MPEG-2 Video in a Routed IP Network : Transport stream errors and their effects on video quality. Proc. of $6^{\text {th }}$ workshop on Interactive Distributed Multimedia System, Toulouse, France (1999)

4. Clark, D., Tennenhouse, D.: Architectural Considerations for a New Generation of Protocols. Proceedings of the ACM SIGCOMM'90, Philadelphia, USA (1990)

5. Dempsey, B.J., Liebeherr, J., Weaver, A.C.: On retransmission-based Error Control for Continuous Media Traffic in Packet-Switching Networks. Computer Networks and ISDN Systems, vol. 28, no. 5 (1996)

6. Dwyer, D., Ha, S., Li, J.R., Bharghavan, V.: An Adaptive Transport Protocol for Multimedia Communication. Proc. of IEEE Int. Conference on Multimedia Computing and Systems, Austin (1998)

7. Gong, F., Parulkar, G.: An Application-Oriented Error Control Scheme for High-Speed Networks. IEEE/ACM Transactions on Networking, vol. 4, no. 5 (1996)

8. Grinnemo, K., Brunstrom, A.: Evaluation of the QoS offered by PRTP-ECN - A TCPCompliant Partially Reliable Transport Protocol. Proc. of $9^{\text {th }}$ Int. Workshop on Quality of Service IWQoS 2001, Karlsruhe, Germany (2001)

9. Hehmann, D.B., Salmony, M.G., Stüttgen, H.J.: Transport services for multimedia applications on broadband networks. Computer Communication, vol. 13, no. 4 (1990)

10. Hoffman, et al: RFC 2250 : RTP Format for MPEG1/MPEG2 Video. Internet Draft (1998)

11. ISO/IEC 11172-2: Coding of moving pictures and associated audio for digital storage media at up to about $1.5 \mathrm{Mbit} / \mathrm{s}$.

12. Karlsson, G.: Asynchronous transfer of video. IEEE Computer Magazine, vol. 34, no. 8 (1996)

13. Lecuire, V., Lepage, F.: Contrôle déterministe de la fiabilité par un marquage direct sur les paquets. Electronic Journal on Networks and Distributed Processing, vol. 9 (2000)

14. Le Gall, D.: MPEG : A video compression standard for multimedia applications. Communications of the ACM, vol. 34, no. 4 (1991)

15. Leicher, C.: Hierarchical Encoding of MPEG Sequences Using Priority Encoding Transmission (PET). Tech. Report TR-94-058, ICSI, Berkeley (1994)

16. Marasli, M., Amer, P., Conrad, P.: Retransmission-based Partial-Reliable Transport Service : An Analytic Model. Proc. of the IEEE Infocom'96, San Francisco (1996)

17. Moon, S.B., Kurose, J., Towsley, D.: Packet Audio Playout Delay Adjustement : Performance Bounds and Algorithms. ACM/Springer Multimedia Systems, vol. 6, no. 1 (1998)

18. Pillai, R.R., Prabhakaran, B.P., Qiang, Q.: A Forward Error Recovery Technique For MPEG-II Video Transport. Proc. of $7^{\text {th }}$ ACM Int. Multimedia Conference, Orlando (1999)

19. Rasheed, Y., Leon-Garcia, A.: AAL 1 with FEC for the Transport of CBR MPEG2 Video over ATM Networks. Proc. of IEEE Infocom'96, San Francisco (1996)

20. Rojas-Cardenas, L., Dairaine, L., Senac, P., Diaz, M.: Error recovery mechanisms based on retransmissions for video coded with motion compensation techniques. Proc. of IEEE Packet Video Workshop, New York (1999) 